\title{
Investigation of microemulsion system for transdermal delivery of ligustrazine phosphate
}

\author{
Ying Cui ${ }^{1,2}$, Lingzhi $\mathrm{Li}^{1,2}$, Jun Gu${ }^{1}$, Ting Zhang ${ }^{3 \star}$ and Li Zhang ${ }^{1}$ \\ ${ }^{1}$ Department of Medicine Chemistry, Logistics University of Chinese People's Armed Police Forces, Tianjin 300162, \\ P. R. China. \\ ${ }^{2}$ Tianjin Key Laboratory of Occupational and Environmental Hazards Biomarkers, Tianjin 300162, P. R. China. \\ ${ }^{3}$ Department of Pharmacy, Xiangya Hospital, Central South University, Changsha 410008, P. R. China.
}

Accepted 30 September, 2011

\begin{abstract}
Ligustrazine phosphate is commonly used as an efficient drug to treat a variety of cardiovascular disorders with frequent administration for a short biological half-life. The purpose of the present study was to investigate a microemulsion system for transdermal delivery of ligustrazine phosphate for the convenient, efficient and safe administration. The existence regions of microemulsions containing isopropyl myristate, labrasol, plurol oleique ${ }^{\circledR}$ and water were investigated in pseudo-ternary phase diagrams, according to which microemulsion vehicles were prepared to observe average diameter, $\mathrm{pH}$ value, stability and evaluate respective effect of each component on percutaneous delivery in vitro. The prepared microemulsions had average diameters ranging from 32.1 to $108.7 \mathrm{~nm}$, mild $\mathrm{pH}$ values and suitable stability. The optimized microemulsion with permeation flux of $41.01 \mu \mathrm{g} / \mathrm{cm}^{2} / \mathrm{h}$ across rat skin in vitro, showed no obvious irritation on back skin of rabbits. The results indicated that the studied microemulsion system might be a promising vehicle for transdermal delivery of ligustrazine phosphate.
\end{abstract}

Key words: Microemulsion, transdermal delivery, ligustrazine phosphate.

\section{INTRODUCTION}

Ligustrazine phosphate (LP) has been reported to be able to inhibit platelet aggregation in vitro and lower blood pressure through vasodilation, and is used as an efficient drug to treat a variety of cardiovascular disorders. The component has a short biological half-life of about $0.167 \mathrm{~h}$ which necessitates frequent administration (Chen and Liu, 2004; Li, 2005). Transdermal delivery has many advantages over oral route and venous administration. It could avoid first pass effect, the administration is convenient and simple for patients, and it has the possibility of immediate withdrawal of the treatment whenever necessary. Despite the great potential of transdermal delivery, only a few drug formulations are available commercially. The main reason is the barrier function of human skin that is considered to be the most impermeable for drug. The outermost layer of the skin, the

*Corresponding author. E-mail: laozhang_fresh@yahoo.com.cn. Tel: 86-0731-84327460.
Stratum corneum (SC), is the principal rate limitation to percutaneous delivery (Pilgram et al., 1998; Moghimi et al., 1996).

Microemulsion is a clear, thermodynamically stable, isotropic dispersion mixture consisting of oil, water, surfactant and cosurfactant, which has typically a droplet diameter of approximately $100 \mathrm{~nm}$ or less (Kogan and Garti, 2006). The advantages of microemulsion on transdermal delivery have been proposed to be mechanisms as following. Firstly, the high solubility potential for both lipophilic and hydrophilic drugs in microemulsion systems could increase drug diffusion ability towards skin (Yuan et al., 2006; Changez et al., 2006); Secondly, ingredients of microemulsion (oil, surfactant and cosurfactant) acting as permeation enhancers, could destroy the barrier structure of SC and enhance the permeation flux of drug across skin (Delgado-Charro et al., 1997; Dreher et al., 1997). Because of the special structure of microemulsion, it also could increase stability of some drugs (Polona et al., 2003). Microemulsions have been subjected to numerous 
Table 1. Compositions of the selected microemulsion formulations.

\begin{tabular}{lcccccccc}
\hline Component (\%) & Control & ME1 & ME2 & ME3 & ME4 & ME5 & ME6 & ME7 \\
\hline IPM & 0 & 51.50 & 48.15 & 45.22 & 56.50 & 62.34 & 55.03 & 58.28 \\
Labrasol & 0 & 17.15 & 16.05 & 15.08 & 13.95 & 10.23 & 15.79 & 14.63 \\
Plurol oleique $^{\circledR}$ & 0 & 17.15 & 16.05 & 15.08 & 13.95 & 10.23 & 15.79 & 14.63 \\
Water & 99.5 & 13.70 & 19.25 & 24.12 & 15.10 & 16.70 & 12.89 & 11.96 \\
LP & 0.5 & 0.5 & 0.5 & 0.5 & 0.5 & 0.5 & 0.5 & 0.5 \\
\hline
\end{tabular}

drugs during the last decades, because of their great potential in transdermal delivery (Peira et al., 2001; Lee et al., 2005; Escribano et al., 2003).

In spite of numerous advantages on transdermal delivery, microemulsion often requires a high content of surfactant, which could cause skin irritation (Lawrence and Rees, 2000). There have been studies concerning low-irritant caprylocaproyl macrogolglycerides based microemulsions as drug delivery vehicles for topical application (Djordjevic et al., 2004; Kreilgaard, 2001). Application of cosurfactant could decrease the amount of surfactant. Short- or medium-chain alcohols have been reported as pharmaceutical unacceptable cosurfactant for their skin irritation. The low-irritation surfactants with structure of polyglycerol fatty acid ester have been used as cosurfactant in combination with caprylocaproyl macrogolglyceride in microemulsions (Kumar and Mittal, 1999; Baroli et al., 2000). Isopropyl myristate (IPM) is commonly used in cosmetics and is well-tolerated. It has been used as the oil phase in microemulsion producing no irritation on albino mice (Gupta et al., 2005). It has been reported that caprylocaproyl macrogolglycerides with IPM as oil phase could form large microemulsion area in their phase diagram (Choi et al., 1997).

The objective of this study was to find an efficient, stable and low-irritant microemulsion vehicle for transdermal delivery of LP which was composed of IPM, labrasol (PEG-8 caprylic/capric glycerides), plurol oleique $^{\circledR}$ (polyglyceryl-6 dioleate) and water. Low concentration of surfactant and cosurfactant were applied in formula design for the purpose of low irritation, and the relative high concentration of water was selected in formula for drug load potential of microemulsion for further investigation.

\section{MATERIALS AND METHODS}

Labrasol and plurol oleique ${ }^{\circledR}$ were kindly donated by Gattefosse (France). IPM was procured from Kangkede Regent Company (China). LP was obtained from Beijing Double-crane Pharmaceutical Co., Ltd. (China). Water was deionized and filtered in our lab. Methanol was high performance liquid chromatography (HPLC) grade. The other chemical solvents were analytical grade and were used without further purification.

\section{Construction of phase diagrams}

In order to find out the concentration range of components for the existence range of microemulsions, pseudo-ternary phase diagrams were constructed using titration method at $25^{\circ} \mathrm{C}$. Phase diagrams were prepared with 1:2, 1:1 and 2:1 weigh ratios of labrasol to plurol oleique $^{\circledR}$, respectively. At each ratio of surfactant to cosurfactant, the ratio of oil to surfactant mixture (surfactant and cosurfactant) was varied as $1: 9,2: 8,3: 7,4: 6,5: 5,6: 4,7: 3,8: 2$ and 9:1. Water was added drop by drop into oily mixture (oil, surfactant and cosurfactant) under gentle magnetic stirring. After being equilibrated, the mixtures were assessed visually and determined as being microemulsion or crude emulsion (Zhao et al., 2006).

\section{Preparation of drug-load microemulsion}

According to the microemulsion regions in the phase diagrams, the microemulsion formulations were selected at different component ratios as described in Table 1 . In order to prepare the drug-load microemulsion, appropriate amount of LP was dissolved in water and calculated amount of IPM and surfactant mixture were mixed. The transparent drug-load microemulsion was prepared by adding the solution of LP to the oily mixture obtained earlier under gentle magnetic stirring. In the designed formula, the same ratio of IPM to surfactant mixture was shared by ME1, ME2 and ME3; the same ratio of IPM to water was shared by ME1, ME4 and ME5; and the same ratio of water to surfactant mixture was shared by ME1, ME6 and ME7. Miscibility test using water-soluble methylene blue and fat-soluble Sudan III was conducted to determine the type of microemulsions after preparation.

The aqueous solution containing $0.5 \%$ of LP was prepared as the control.

\section{Determination of the solubility of LP in microemulsion}

An excess amount of LP was added to each of the microemulsion vehicles and shaken at $32^{\circ} \mathrm{C}$ for $72 \mathrm{~h}$. The suspension was filtered through a membrane filter $(0.45 \mu \mathrm{m})$, and the concentration of LP in the filtrate was determined by HPLC.

\section{Characterization and stability of microemulsion}

The average droplet size of prepared microemulsion was checked by dynamic light scattering using a Zetasizer Nano ZS (Malvern Instruments, England) at $25^{\circ} \mathrm{C}$. The $\mathrm{pH}$ value of microemulsion was determined at $25^{\circ} \mathrm{C}$ using a pHS-3C digital acidimeter (Shanghai REX Instrument Factory, China).

To characterize the conductivity of microemulsion, a DDS-IIC conductivity meter (Shanghai Precision and Scientific Instrument, China) was used at $25^{\circ} \mathrm{C}$.

The viscosity of microemulsion was measured at $25^{\circ} \mathrm{C}$ using a NDJ-8S digital viscometer (Shanghai Precision and Scientific Instrument, China) with a No. 1 rotor set at $30 \mathrm{rpm}$.

Centrifuge tests were carried out to assess the physical stability of microemulsion. Microemulsion vehicles were centrifuged for 30 min 
at 13,000 rpm and $4 \mathrm{~h}$ at $4000 \mathrm{rpm}$ (Zhao et al., 2006).

Microemulsion vehicles were stored at 5,25 and $32{ }^{\circ} \mathrm{C}$ for 6 months. Then, the clarity, phase separation and concentration of LP were investigated monthly.

\section{In vitro skin permeation study}

Animal experiment was carried out in accordance with the National Institute of Health (NIH) guidelines, and with institutional review board approval. The skins were obtained from the abdominal surface of Wistar rats with weight of $180 \pm 20 \mathrm{~g}$. After hairs were removed with a clipper, the skins were excised and the extraneous tissues were trimmed surgically. The excised rat skins were washed carefully, and then wrapped between aluminum foils and stored at about $-20^{\circ} \mathrm{C}$ prior to use (less than 2 weeks).

Franz diffusion cells with effective diffusion area of $2.86 \mathrm{~cm}^{2}$ were used in the permeation studies. The skin previously rinsed with normal saline was sandwiched between the cells with the SC facing the donor compartment. The maximum capacity of donor and receiver compartment was 2 and $6 \mathrm{ml}$, respectively. The donor compartment contained $1.5 \mathrm{ml}$ of microemulsion, and the receiver compartment was filled with $6 \mathrm{ml}$ of phosphate buffered saline $(\mathrm{pH}$ 7.4). The cells were maintained at $32 \pm 0.5^{\circ} \mathrm{C}$ and covered with parafilm to prevent evaporation of water. The contents of the receiver compartment were stirred with a magnetic bar at speed of $100 \mathrm{rpm}$. At appropriate time, $0.5 \mathrm{ml}$ of sample was withdrawn from the receiver compartment for HPLC determination and an equivalent amount of phosphate buffered saline $(0.5 \mathrm{ml})$ was added immediately to the receiver compartment to maintain a constant volume.

The cumulative amount of LP permeated through excised rat skins was calculated by Equation 1:

$Q_{n}=\frac{V C_{n}+\sum_{i=1}^{n-1} C_{i} V_{i}}{A}$

Where $C_{n}$ was the drug concentration of the receiver solution at each sampling time, $C_{i}$ was the drug concentration of the sample, and $V$ and $V_{i}$ were the volumes of the receiver solution and the sample, respectively. $A$ was the effective diffusion area $(A=2.86$ $\mathrm{cm}^{2}$ ). The cumulative amount of LP permeated per unit skin surface area was plotted against time; the slope of the linear portion of the plot was calculated as steady state flux $\left(J_{s s}\right)$. The permeability coefficient $K_{p}$ was calculated as Equation 2, where $C_{0}$ was the concentration of drug in vehicle.

$$
K_{p}=J_{s s} / C_{o}
$$

\section{HPLC analysis of LP}

Samples were analyzed by HPLC method. The analytical column was Shimadzu shimpack $\mathrm{C}_{18}(5 \mu \mathrm{m}, 4.6 \times 250 \mathrm{~mm})$. The mobile phase was methanol-water $(60: 40)$ at the flow rate of $1.0 \mathrm{ml} / \mathrm{min}$, and the detection wavelength was set at $285 \mathrm{~nm}$. The assay was linear in the concentration range of 0.1 to $10 \mu \mathrm{g} / \mathrm{ml}$. The recovery rate ranged from 98.6 to $101.5 \%$. The relative standard deviation (RSD) value for precision was below $0.78 \%$. All operations were carried out at ambient temperature.

\section{Statistical analysis}

All skin permeation experiments were repeated three times and data were expressed as the mean value \pm SD. Statistical data were analyzed by one-way analysis of variance (ANOVA), and a P-value of 0.01 was considered to be significant.

\section{Skin irritation study}

The optimized microemulsion and the control solution were selected as the test formulations for skin irritation studies. Samples were applied to the shaved dorsal skins of five New Zealand rabbits for 7 days. The painted sites were covered with gauze fixed with sticking plaster, and observed and evaluated for sign of erythema or oedema daily (Jain et al., 1996).

\section{RESULTS AND DISCUSSION}

\section{Phase study}

The construction of pseudo-ternary phase diagrams was used to determine the concentration range of components in the existence range of microemulsion. The studied system was composed of safe constituents including IPM, labrasol, plurol oleique ${ }^{\circledR}$ and water. Pseudo-ternary phase diagrams at three different ratios of labrasol/plurol oleique $^{\circledR}$ were shown in Figure 1. The translucent microemulsion region was presented as ME in the phase diagrams. The rest of the region on the phase diagram represented the turbid and conventional emulsion (crude emulsion) determined by visual observation. The labrasol/plurol oleique ${ }^{\circledR}$ ratio change significantly influenced the microemulsion area. When the weight ratio of labrasol to plurol oleique ${ }^{\circledR}(\mathrm{Km})$ was $1: 1$, the microemulsion area in diagram was the maximum. It has been reported that at the optimum $\mathrm{Km}$ value, cosurfactant molecules could exactly insert into the cavities between surfactant molecules (Kawakami et al., 2002).

\section{Preparation of microemulsion}

In Vandamme (2002) study, the order of the addition of surfactant and cosurfactant has significant influence on the preparation of microemulsions, which have low concentrations of surfactant and cosurfactant. Given that there was low concentration of surfactant mixture in the designed formulas, the influence of the components on the preparation of microemulsion was studied. In the investigation, labrasol and plurol oleique ${ }^{\circledR}$ were added together or alone to oily phase or aqueous phase before the two phases were mixed. And it proved no effect on the equilibrium time. It might be due to quick distribution of the surfactant and cosurfactant between oily phase and aqueous phase (Chen et al., 2004). And all the microemulsions were judged as type of water in oil (W/O) by miscibility test.

\section{Solubility study}

The solubility of LP in microemulsion vehicle is as shown 

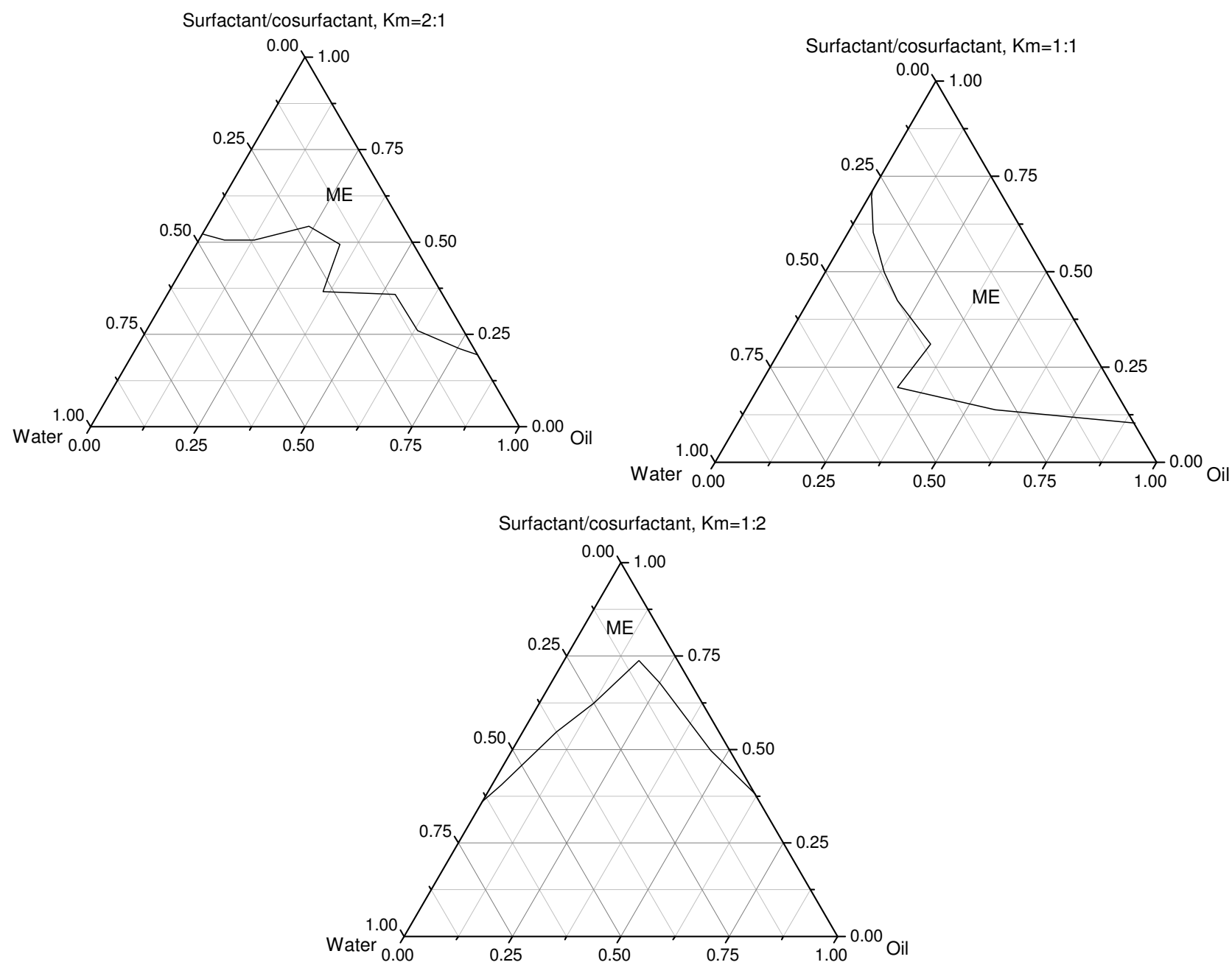

Figure 1. Pseudo-ternary phase diagrams of microemulsion (ME) composed of IPM, labrasol/plurol oleique ${ }^{\circledR}$ and water.

in Table 2. The decrease of solubility was not proportional to the decrease of water amount. It could be mainly explained as the addition of surfactant mixture in microemulsion. In fact, part of surfactant mixture could participate in both oil and water phase for its amphiphilic character, which could increase the solubility of drug in these phases (Delgado-Charro et al., 1997).

\section{Characterization and stability of microemulsion}

It was observed that the average diameters of microemulsion vehicles ranged from 32.1 to $108.7 \mathrm{~nm}$ (Table 2). ME1, ME2 and ME3 shared the fixed ratio of oil to surfactant mixture; the droplet size of the microemulsion was increased with the increased water amount. It has been reported that small size drop produces more surface tension, and the depression needs more surfactant mixture (Yuan et al., 2006). ME1, ME4 and ME5 had the same oil/water ratio; the average diameter of the microemulsion was increased with the decreased surfactant mixture amount. It could also be explained as surface tension depression with surfactant mixture concentration increase. ME6 and ME7 had the same water/surfactant mixture ratio with ME1. With a little difference of IPM amount, ME6 had a similar diameter with ME1. In comparison with ME1, the increased IPM amount of ME7 could decreased the concentration of surfactant mixture locating at the interface of oil and water, and the mean diameter of $45.3 \mathrm{~nm}$ of ME7 was observed.

The $\mathrm{pH}$ values of microemulsion vehicles were in a range of 6.51 and 6.94 (Table 2), which was attributed to the fact that the organic components involved in the microemulsions were ester structure except the weak acid character of LP. The $\mathrm{pH}$ value within the physiological range is one of the important conditions for low-irritation vehicle.

The conductivity and viscosity results were showed in Table 2. Conductivity measurement is the most frequently used technique to understand the microstructure and 
Table 2. Mean diameter, pH value, viscosity, conductivity, solubility and permeation parameters of the selected microemulsion formulations.

\begin{tabular}{lccccccc}
\hline Vehicle & $\begin{array}{c}\text { Mean diameter } \\
(\mathbf{n m})\end{array}$ & $\mathbf{p H}$ & $\begin{array}{c}\text { Viscosity } \\
(\mathbf{m P a} \cdot \mathbf{s})\end{array}$ & $\begin{array}{c}\text { Conductivity } \\
(\boldsymbol{\mu S} / \mathbf{c m})\end{array}$ & $\begin{array}{c}\text { Solubility } \\
(\mathbf{m g} / \mathbf{g})\end{array}$ & $\begin{array}{c}\mathbf{J}_{\mathbf{s s}} \\
(\boldsymbol{\mu g} / \mathbf{c m} / \mathbf{h})\end{array}$ & $\begin{array}{c}\mathbf{K}_{\mathbf{p}} \\
\left(\times \mathbf{1 0} \mathbf{0}^{-3} \mathbf{c m} / \mathbf{h}\right)\end{array}$ \\
\hline ME1 & $32.1 \pm 1.2$ & $6.51 \pm 0.03$ & $150.4 \pm 0.1$ & $7.9 \pm 0.2$ & $25.32 \pm 1.22^{*}$ & $31.08 \pm 3.88^{*}$ & $4.04 \pm 0.50^{*}$ \\
ME2 & $46.9 \pm 4.1$ & $6.75 \pm 0.02$ & $155.6 \pm 0.0$ & $14.6 \pm 0.6$ & $29.06 \pm 0.77^{*}$ & $24.28 \pm 4.29^{*}$ & $3.16 \pm 0.56^{*}$ \\
ME3 & $99.1 \pm 5.0$ & $6.89 \pm 0.03$ & $209.0 \pm 0.0$ & $32.3 \pm 1.0$ & $32.11 \pm 1.93^{*}$ & $10.93 \pm 2.39^{*}$ & $1.42 \pm 0.31^{*}$ \\
ME4 & $45.6 \pm 4.5$ & $6.83 \pm 0.05$ & $150.8 \pm 0.1$ & $9.3 \pm 0.4$ & $21.86 \pm 0.91^{*}$ & $11.22 \pm 2.33^{*}$ & $1.46 \pm 0.31^{*}$ \\
ME5 & $108.7 \pm 9.7$ & $6.94 \pm 0.04$ & $155.2 \pm 0.0$ & $10.9 \pm 1.9$ & $18.15 \pm 0.40^{*}$ & $21.95 \pm 3.64^{*}$ & $2.85 \pm 0.47^{*}$ \\
ME6 & $36.5 \pm 2.8$ & $6.77 \pm 0.04$ & $145.0 \pm 0.0$ & $7.3 \pm 0.7$ & $22.44 \pm 1.73^{*}$ & $41.01 \pm 3.43^{*}$ & $5.32 \pm 0.45^{*}$ \\
ME7 & $45.3 \pm 2.2$ & $6.85 \pm 0.03$ & $144.3 \pm 0.0$ & $7.3 \pm 0.9$ & $19.49 \pm 1.02^{*}$ & $24.18 \pm 4.30^{*}$ & $3.14 \pm 0.56^{*}$ \\
control & - & - & - & - & $65.52 \pm 1.56$ & $1.39 \pm 0.45$ & $0.28 \pm 0.09$ \\
\hline
\end{tabular}

*Significantly different from the control $(P<0.01)$.

structural changes of selected microemulsions (Boonme et al., 2006). In the region of low water content, water in oil microemulsion is formed that has a high resistance to conductivity. The low conductivity values reveal the W/O structure of microemulsions. The conductivity increased with the increase amount of water. It is well known that the increasing of volume fraction of dispersed phase in microemulsions brings to the increase of viscosity (Bennett et al., 1982). In W/O microemulsion, it might be the consequence of attractive interaction and aggregation of droplets of water phase. The results of viscosity (Table 2) confirmed the conclusion.

In centrifuge tests, microemulsions were stable except ME3 and ME5 in which phase separation or turbid was observed. The components of the two formulas neared the margin of microemulsion area in the phase diagram. It has been reported that the internal phase is ready for temporary aggregation when microemulsion component is near the margin of microemulsion area (Kreilgaard, 2001), and the microemulsions are recovered by mild shake.

Microemulsions stored at 25 and $32^{\circ} \mathrm{C}$ showed no changes in clarity, phase behavior and degradation of LP, while at $5^{\circ} \mathrm{C}$, turbidity happened in ME2, ME3 and ME5. According to the previous report (Chen et al., 2004), the coagulating of the internal phase at low temperature might lead to turbidity, and these microemulsions are recovered by heating up.

\section{In vitro skin permeation study}

\section{Effect of water content on permeation of LP through rat skins from microemulsions with same oil/surfactant mixture ratio}

In formula ME1, ME2 and ME3, the content of water was increased in order and the oil/surfactant mixture ratio was fixed. As the content of water was increased, the permeation of drug through skin was decreased significantly (Table 2 and Figure 2).

It has been reported that the permeation of 5-fluorouracil increased significantly with the increase of water concentration in a water in oil (W/O) microemulsion (Gupta et al., 2005). It might be due to the increase of hydration level of SC and fluidization of lipid alkyl chain in SC, which could facilitate the permeation of drug through skin, but in our studied microemulsions (IPM/labrasol/plurol oleique ${ }^{\circledR} /$ water), the transdermal permeation of LP decreases with the increase of water concentration. LP had high solubility in water, and with the increase amount of water, the solubility of LP in microemulsion increases. As a result, the thermodynamic activity of LP in vehicle might decrease, which is an important driving force for drug release and penetration into skin, and it reflects the relative activities of drug in different phase.

The drug in microemulsion could penetrate into skin in the form of microemulsion droplet. With the result shown in Table 2, the mean diameter increase was found in order of ME1, ME2 and ME3, which could be attributed to addition of water. Small droplet is preferred in the term of skin penetration. Therefore, with the increase of water amount, the transdermal permeation of LP decreases. This finding was consistent with a previous document (Sintov and Shapiro, 2004).

In another aspect, the decrease concentration of surfactant mixture and IPM which could destroy the structure of SC (described in details subsequently) was considered as a factor resulting in transdermal permeation decrease of ME2 and ME3.

\section{Effect of surfactant mixture content on permeation of LP through rat skins from microemulsions with same oil/water ratio}

In formula ME1, ME4 and ME5, the content of surfactant mixture was decreased in order, and the ratio of oil to water was fixed. It was apparent that as the content of surfactant mixture was increased, the permeation flux of LP was found to decrease firstly (ME5/ME4) and then increase (ME4/ME1) (Table 2 and Figure 3). 


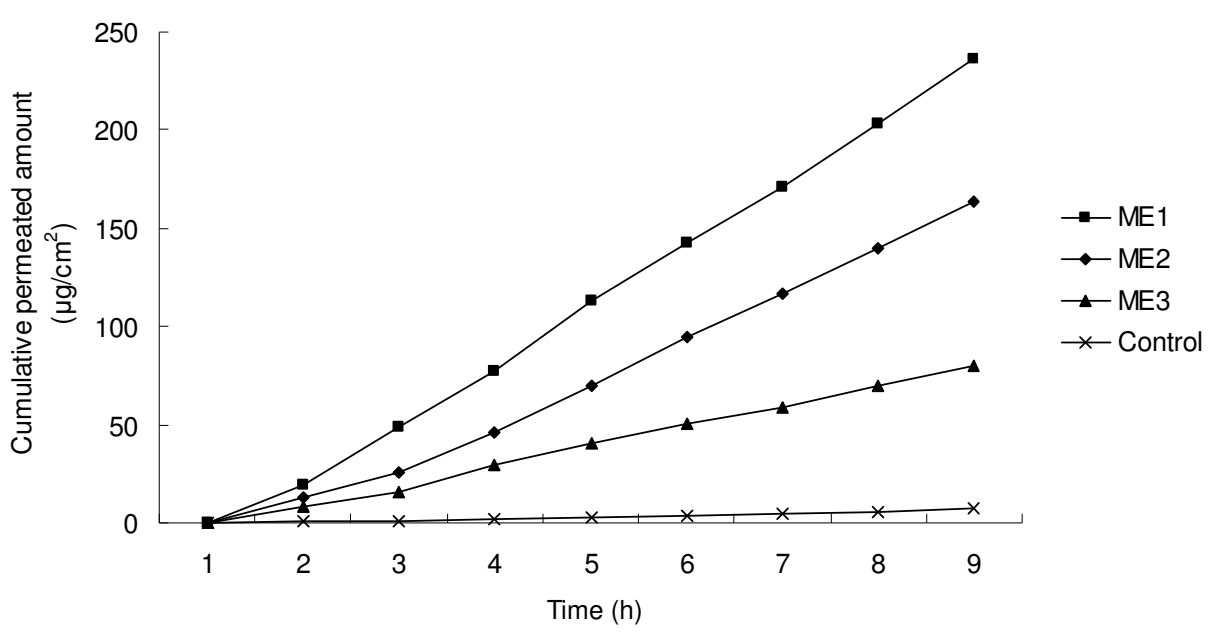

Figure 2. Permeation profiles of LP through rat skins from the microemulsions with same oil/surfactant mixture ratio.

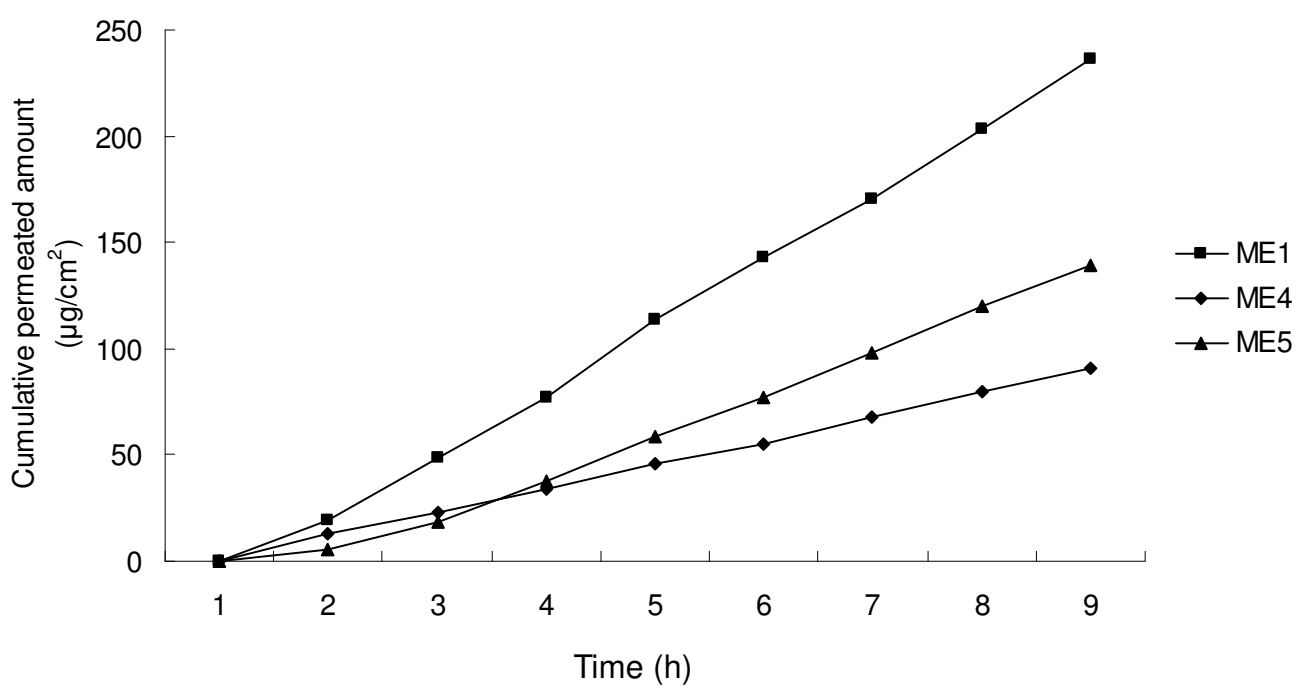

Figure 3. Permeation profiles of LP through rat skins from the microemulsions with same oil/water ratio.

In this W/O microemulsion, water was the internal phase in which LP is mainly located and IPM was the external phase. With the increase of surfactant mixture concentration, the thickness of the interface of "oil and water" might be increased and it could hinder the diffusion of LP from the internal phase to the external phase. Except that, as the content of surfactant mixture was increased, the solubility of LP in microemulsion increases, resulting in the decrease of the thermodynamic activity of LP in vehicle. The reasons aforementioned description might mainly contribute to the permeation decrease of ME4 in comparison with ME5.

It has been reported that monomer surfactants (or oil) could diffuse to skin surface and act as enhancers, either by disrupting the lipid structure of SC to facilitate diffusion through the barrier phase, or by increasing the solubility of drug in skin to improve the partition coefficient of the drug between the skin and the vehicle (Kreilgaard, 2002). The increase of surfactant mixture concentration could enlarge the structure destruction of SC to facilitate permeation across the skin. In addition to that, surfactant mixture could locate in both oil and water phase for its amphiphilic character, thus drug dissolved in external phase could be improved with the concentration increase of surfactant mixture in this phase (Delgado-Charro et al., 1997). With the increase of surfactant mixture amount in formula, the concentration of surfactant mixture and LP in IPM might increase, resulting in the increase of the concentration gradient between skin and the external phase. Therefore, the SC destruction and LP concentration increase in 


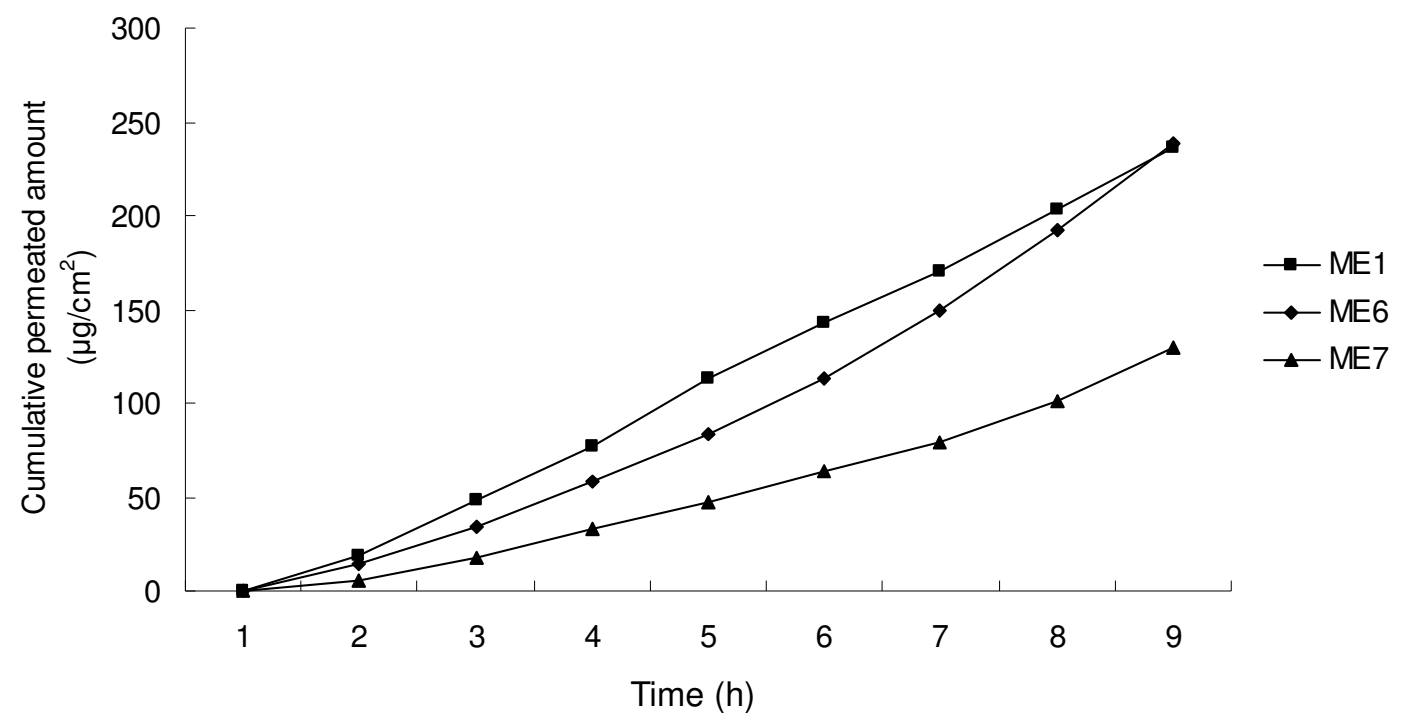

Figure 4. Permeation profiles of LP through rat skins from the microemulsions with same water/surfactant mixture ratio.

external phase might mainly contribute to the permeation enhancement of ME1 in comparison with ME4.

\section{Effect of oil content on permeation of LP through rat skins from microemulsions with same water/surfactant mixture ratio}

In formula ME1, ME6 and ME7, the content of IPM was increased in order and the ratio of water to surfactant mixture was fixed. The increase of IPM amount caused the increase of permeation flux firstly (ME1/ME6), then decrease of it (ME6/ME7) (Table 2 and Figure 4).

As a routine transdermal enhancer, IPM is commonly used in skin preparations and is well-tolerated. It has been suggested that an ideal enhancer should have a solubility control parameter similar to skin, and IPM just has the close value with SC (Sloan et al., 1986). The increase of IPM concentration could decrease skin barrier character through SC disturbance. Except that, with the increase of oil amount, the solubility of LP in microemulsion decreases, resulting in the increase of the thermodynamic activity of LP in vehicle. In another aspect, the increase of IPM concentration might depress the interaction between water phase and SC which is an important factor influencing the partition of water soluble drug into the skin, and with the increase of IPM amount in formula, the concentration of surfactant mixture and LP in IPM might decrease, resulting in the decrease of the concentration gradient between IPM and the skin. The increased amount of IPM also caused the increase of droplet size of ME7 in comparison with ME1 and ME6. As a result, with the increase of IPM amount from 51.50 (ME1) to $55.03 \%$ (ME6), the increase of permeation flux might be mainly influenced by SC barrier depression and thermodynamic activity increase of LP. Further increase of IPM concentration (ME6/ME7), the decrease of permeation flux might be mainly attributed to SC hydration depression, concentration gradient decrease and droplet size increase.

In combination consideration of stability and transdermal permeation, ME6 was selected as the optimum microemulsion formula in percutaneous delivery of LP.

\section{Skin irritation study}

The control solution caused no syndrome of irritation. Topical application of ME6 showed no erythema or oedema on the back skins of five rabbits in seven days. Rubefaction was observed only on one rabbit from the third day, and disappeared quickly at the seventh day as soon as ME6 was removed from the back skin.

\section{Conclusion}

A microemulsion system composed of IPM, labrasol, plurol oleique ${ }^{\circledR}$ and water, was developed and evaluated as a potential transdermal delivery system for LP. The prepared microemulsions had average diameters ranging from 32.1 to $108.7 \mathrm{~nm}$, mild $\mathrm{pH}$ values for the reason of component selection and suitable stability. The microemulsions could increase the permeation rate of LP 7.86 to 29.50 times when compared with the control. The optimum microemulsion formula (ME6) exhibited a high permeation rate of $41.01 \mathrm{\mu g} / \mathrm{cm}^{2} / \mathrm{h}$ across rat skins in vitro and showed no obvious skin irritation on the back skin of rabbits. These results showed that the studied microemulsion system might be a promising vehicle for the percutaneous delivery of LP. 


\section{REFERENCES}

Baroli B, Lopez-Quintela MA, Delgado-Charro MB, Fadda AM, Blanco-Méndez J (2000). Microemulsions for topical delivery of 8-methoxsalen. J. Control Release, 69(1): 209-218.

Bennett KE, Hatfield JC, Davis HT, Macosko CW, Scriven LE (1982). Viscosity and conductivity of microemulsions. In: Robb ID editor. Microemulsions. Plenum Press, New York, pp. 65-84.

Boonme P, Krauel K, Graf A, Rades T, Junyaprasert VB (2006). Characterisation of microstructure formed in isopropyl palmitate/water/Aerosol OT: 1-butanol (2:1) system. Pharmazie, 61(11): 927-932.

Changez M, Varshney M, Chander J, Dinda AK (2006). Effect of the composition of lecithin/n-propanol/isopropyl myristate/water microemulsions on barrier properties of mice skin for transdermal permeation of tetracaine hydrochloride: In vitro. Colloids Surf. B Biointerfaces, 50(1): 18-25.

Chen $\mathrm{H}$, Chang $\mathrm{X}$, Weng $\mathrm{T}$, Zhao X, Gao Z, Yang $\mathrm{Y}, \mathrm{Xu} \mathrm{H}$, Yang X (2004). A study of microemulsion systems for transdermal delivery of triptolide. J. Control Release, 98(3): 427-436.

Chen Y, Liu M (2004). Systemic evaluation of security of ligustrazine for treatment of cerebral infarction. Chin. J. Clin. Rehabil., 8(7): 1299-1301.

Choi E, Jin K, Rhim S, Kim M (1997). Microemulsions with PITs near room temperature. Cosm. Toil., 112: 85-90.

Delgado-Charro MB, Iglesias-Vilas G, Blanco-Mendez J, Lopez-Quintela MA, Marty JP, Guy RH (1997). Delivery of a hydrophilic solute through the skin from novel microemulsion systems. Eur. J. Pharm. Biopharm., 43(1): 37-42.

Djordjevic L, Primorac M, Stupar M, Krajisnik D (2004). Characterization of caprylocaproyl macrogolglycerides based microemulsion drug delivery vehicles for an amphiphilic drug. Int. J. Pharm., 271(1-2): 11-19.

Dreher F, Walde P, Walther P, Wehrli E (1997). Interaction of a lecithin microemulsion gel with human stratum corneum and its effect on transdermal transport. J. Control Release. 45(2): 131-140.

Escribano E, Calpena AC, Queralt J, Obach R, Doménech J (2003). Assessment of diclofenac permeation with different formulations: anti-inflammatory study of a selected formula. Eur. J. Pharm. Sci., 19(4): 203-210.

Gupta RR, Jain SK, Varshney M (2005). AOT water-in-oil microemulsions as a penetration enhancer in transdermal drug delivery of 5-fluorouracil. Colloids Surf. B. Biointerfaces, 41(1): 25-32.

Jain GK, Sharma AK, Agrawal SS (1996). Transdermal controlled administration of verapamil-enhancement of skin permeability. Int. J. Pharm., 130(2): 169-177.

Kawakami K, Yoshikawa T, Moroto Y, Kanaoka E, Takahashi K, Nishihara Y, Masuda K (2002). Microemulsion formulation for enhanced absorption of poorly soluble drugs: 1 . Prescription design. J. Control Release, 81(1-2): 65-74.
Kogan A, Garti N (2006). Microemulsions as transdermal drug delivery vehicles. Adv. Colloid Interface Sci., 123(126): 369-385.

Kreilgaard M (2001). Dermal pharmacokinetics of microemulsion formulations determined by in vivo microdialysis. Pharm. Res., 18(3): 367-373.

Kreilgaard M (2002). Influence of microemulsions on cutaneous drug delivery. Adv. Drug Deliv. Supplement., 1(54): S77-S98.

Kumar P, Mittal KL (1999). Handbook of Microemulsion Science and Technology, Marcel Dekker Inc., New York, pp. 323-358.

Lawrence MJ, Rees GD (2000). Microemulsion-based media as novel drug delivery systems. Adv. Drug Deliv. Rev., 45(1): 89-121.

Lee J, Lee Y, Kim J, Yoon M, Choi Y (2005). Formulation of microemulsion systems for transdermal delivery of aceclofenac. Arch. Pharm. Res., 9(28): 1097-1102.

Li P (2005). Pharmacokinetic study and clinical application of ligustrazine hydrochloride. Mod. J. Int. Tradit. Chin. West. Med., 14(12): 1622-1623.

Moghimi HR, Williams AC, Barry BW (1996). A lamellar matrix model for stratum corneum intercellular lipids. II. Effect of geometry of the stratum corneum on permeation of model drugs 5-fluorouracil and oestradiol. Int. J. Pharm., 131(2): 117-129.

Peira E, Scolari P, Gasco MR (2001). Transdermal permeation of apomorphine through hairless mouse skin from microemulsions. Int. J. Pharm., 226(1-2): 47-51.

Pilgram GS, Van Pelt AM, Spies F, Bouwstra JA, Koerten HK (1998). Cryo-electron diffraction as a tool to study local variations in the lipid organization of human stratum corneum. J. Microsc., 189(1): 71-78.

Polona S, Miha H, Andreja ZV, Mirjana G (2003). Sodium ascorbyl phosphate in topical microemulsions. Int. J. Pharm., 256(1-2): 65-73.

Sintov AC, Shapiro L (2004). New microemulsion vehicle facilitates percutaneous penetration in vitro and cutaneous drug bioavailability in vivo. J. Control. Release, 95(2): 173-183.

Sloan KB, Siver KG, Koch SA (1986). The effect of vehicle on the diffusion of salicylic acid through hairless mouse skin. J. Pharm. Sci., 75(8): 744-749.

Vandamme TF (2002). Microemulsions as ocular drug delivery systems: recent developments and future challenges. Prog. Retin. Eye Res., 21(1): 15-34.

Yuan Y, Li S, Mo F, Zhong D (2006). Investigation of microemulsion system for transdermal delivery of meloxicam. Int. J. Pharm., 321(1-2): 117-123.

Zhao X, Liu J, Zhang X, Li Y (2006). Enhancement of transdermal delivery of theophylline using microemulsion vehicle. Int. J. Pharm., 327(1-2): 58-64. 